\title{
Game for Health Professional Education
}

\author{
Alice J. Lin, Michael Chen, and Fuhua (Frank) Cheng
}

\begin{abstract}
Learning medicine is a difficult task that requires both memorization of clinical knowledge as well as practice of medical procedures. As most procedures can cause harm to the patient, it is sometimes not suitable for new students to practice such procedures on actual patients. The challenge, therefore, is to develop new methods of teaching students while minimizing the risk to the patient. Fortunately, modern technology has the means to inject much needed interest into the subject and allows for multiple new formats of learning. As computer games are extremely popular, their role in medical education becomes more and more vital. Serious gaming describes a technology that can educate and train while entertaining users. This paper aims to provide an alternative platform for health science education in a format that appeals to users. A prototype has been developed for the education of health science and it can potentially supplement traditional teaching methods.
\end{abstract}

Index Terms-Medical education, serious games.

\section{INTRODUCTION}

Many recent studies have identified the benefits of using serious video games for a wide range of purposes. Since many games are inexpensive, widely available, and entertaining, combining them with conventional methodologies could provide a powerful means of encouraging people to work more effectively in designated activities. They can be used as a tool to give players a novel way to interact with games in order to develop new skills, promote physical activities, and support social and emotional development. Hence, they have a huge potential as a means of improving achievements in a variety of domains [1]. For a serious game to be successful, an important element is for the game designer to achieve a balance between the fun element and the main purpose of the game. This means that the entertainment element of the game should not be sacrificed in an attempt to reach the main goal of the game. The enjoyment of the game is the very means by which the goal can be reached. Thus, the game should be kept enjoyable, or else the serious goal would not be reached even though it was given the highest attention in the development of the game [2]. Serious games encompass the same goals of "edutainment", but extend far past teaching facts and rote memorization to include all aspects of education: teaching, training, and informing [3].

Many simulation approaches have been developed for the purpose of educating students on the safe delivery of health care. These include the use of mannequins, task trainers, and in situ scenarios. Simulation approaches expose individuals

Manuscript received May 10, 2015; revised August 18, 2015.

Michael Chen and Fuhua (Frank) Cheng are with University of Kentucky, Lexington, KY 40506, USA.

A. J. Lin is with Marshall University, Huntington, WV 25755, USA (e-mail: lina@marshall.edu). and teams in a new way to realistic clinical challenges. A distinguishing feature of simulation and serious games is that it allows participants to experience, in real or near real-time, the consequences of their decisions and actions as they learn new skills and address system anomalies in an environment that does not put patients at risk. Simulations can also be used as a test-bed to identify failure modes and other areas of concern in new clinical processes, procedures, and technologies that might threaten patient safety.

In November 1999, the Institute of Medicine (IOM) estimated that between 44,000 and 98,000 Americans die each year as a result of medical errors [4]. The IOM report quickly elevated awareness of patient safety. Although the United States provides some of the best health care in the world, the number of patients that are harmed is unacceptably high. Traditionally, the education of providers has focused on knowledge acquisition rather than the acquisition of defined levels of acceptable clinical performance. At the same time, it is recognized that many health care procedures are potentially dangerous and very difficult to teach and learn. A troubling paradox of health care delivery is that today's patients can be harmed in the training of tomorrow's practitioners.

Education of healthcare professionals is of utmost importance for patient safety. Education and training must be practiced during the entire working, but it has a high associated cost and sometimes cannot even be achieved. This is because such kinds of training requires human and animal cadavers, human actors, or dolls to allow the practice of certain skills [5]. New technologies bring new possibilities not only to the field of medical training but also to other health professions and could lead to valuable improvements in learning outcomes [6-8].

The apprenticeship model of "see one, do one, teach one" has been reframed as "see one, do many with simulation, teach one" by adopters of simulation and serious game techniques. It is increasingly recognized that the education and training of providers is a life-long process, especially as new technology and less invasive procedures make their way into clinical practice. It is reasonable to expect that simulations benefit not only the inexperienced and vulnerable health care workers at the start of their careers, but also experienced practitioners learning new procedures.

In addition to their promising potential of reducing risks to patients, simulations and serious games are valued for their ability to create conditions that optimize learning. Limited performance repertoires of novices and practitioners learning new procedures can be expanded with a graduated crawl-walk-run approach. Intricate elements of a difficult procedure can be selectively rehearsed again and again. Simulations and serious games can be programmed to provide training on patient complications and equipment malfunctions that rarely occur in practice, but that require decisive action 
and skilled performance when they do occur. Simulations and serious games enable a complex mosaic of multiple skills cognitive, perceptual-motor, and affective - to be simultaneously exercised [9].

\section{BACKGROUND}

While simulation in health care can be traced to the $1960 \mathrm{~s}$ with Resusci-Anne and cardiopulmonary resuscitation training, it was not until the late 1980s that pioneering research efforts started to take hold in anesthesiology with the development of simulation systems that trained participants in clinical management and teamwork [10]. Over the past decade, there has been a progressively increasing interest in a broad array of simulation applications to health care. Advances in medical simulation technology, a progressive lowering of associated costs, a national focus on patient safety, greater recognition of the performance-based component of clinical competency, shortening of hours for residents thereby reducing exposure to less common procedures, and a greater willingness to incorporate lessons learned from other high-risk industries are a few of the factors that help to account for this heightened interest. Other indicators of the heightened interest can be found in the impressive growth of simulation centers in medical and nursing schools.

Serious games or simulators can help medical students significantly improve their skills in performing procedures. The influence of video game experiences on laparoscopic simulator skills has been observed in a few studies that are specifically designed to investigate that goal. Subjects with previous game experience (10 of 25 novice surgeons in training) made significantly less errors on the MIST-VR simulator (Mentice Medical Simulation, Gothenburg, Sweden), even after adjusting for sex and hand dominance [11]. Van Hove et al. followed 35 first-year surgical residents during their first year of training and correlated their initial laparoscopic skill level to trainee characteristics. A history of video game use correlated with significantly higher scores and better skills retention [12].

Simulation games can achieve the high quality outcomes. Simulations create a significantly higher understanding and retention of content than traditional classroom training [13]. Games can be used as an intervention method for therapy, educational, learning, or decision-support [14].

We developed a serious game mainly targeted for medical education, but can also be played by anyone who is interested in the healthcare field.

\section{DESIGN AND DEVELOPMENT}

It is vital that any application be planned well and designed to suit the needs of the healthcare users that it is directed towards. There are many factors that need to be considered, such as usability, accessibility and functionality when developing an application. In the case of a prototype game, it is important that the game be presented in an appealing manner and is also easy to use [15]. The game starts with an introduction in which the player begins learning the game and interaction mode. The activities in the game are interesting and engaging. It can be used as self-directed learning tools and offers the ability to select the level of difficulty in games. The content is reliable with correct flow. Navigation of content is easy and the materials are sufficient and relevant. Players are able to understand the learning goal and control the game while monitoring their own progress.

A variety of levels were built within the prototype game. These levels include the menu and a main body level containing all of the sub-systems. The player will be able to navigate to each sub-system level. Figure 1 shows the architecture of the game. Each new level provides additional challenges, and when a level is successfully completed, the player will get a new score. Upon completion of all levels, the final score will be displayed.

The main aim of this project is to create an alternative solution to traditional medical education by developing an appealing prototype computer game that enables users to learn medicine in a challenging and entertaining way. The information communicated from the computer to the players is both visual and auditory. The interaction of the player with the game is done through traditional interfaces of keyboard, mouse and joystick. The game is three dimensional (3D) and is for a single player. Suitable multimedia elements are especially used. The 3D human models and anatomical systems (skeletal system, nervous system, etc.) can be visualized in all directions.

The design of the levels within the game is important as it contributes directly to the usability of the game and also to general user experience. Initially, the user is presented with an introduction to the game which details the purpose of the prototype and what it is about. After that, the main menu allows the user to view the controls and options, to play the game or close the application. The game provides guidance at each level. Throughout the tutorial, a player is introduced to the controls of the game and the significance of some of the details of the interface. A virtual instructor will appear to give the player hints if the individual makes a mistake. Within the main game levels, information panels will be used to explain the functions of various game parts. Within each game level, the player is not allowed to proceed to the next level until he gives the correct response. The game presents a series of questions with progressive levels of difficulty where the player score is calculated accordingly. When the player runs out of chances, he loses and must start again from the beginning of the last level. The player knows there is a way to win, and he can keep trying until he gets it.

The first level is simple and takes only a relatively small amount of time to complete. It serves as a training step to help the player understand the game's interface and acquire the skills needed to play the game effectively. If the player answers a question correctly, he can go on to the next question. If the answer is incorrect, the virtual instructor will tell him how to find the correct answer or give him a hint.

Level 1 contains main organ systems of the body. The human body is made up of many organ systems that work with one another interdependently. These systems include the integumentary system, skeletal system, muscular system, respiratory system digestive system nervous system, endocrine system cardiovascular system, urinary system, and reproductive systems. The prototype human model includes 
anatomical systems and their related functions. When a user selects some part of the model with a mouse, the related part will be highlighted, showing the details of that part. The model will also include functions for each part. For example, when a user points to the heart, blood circulation will start, or if the user points to the lungs, the lungs will inflate and deflate.

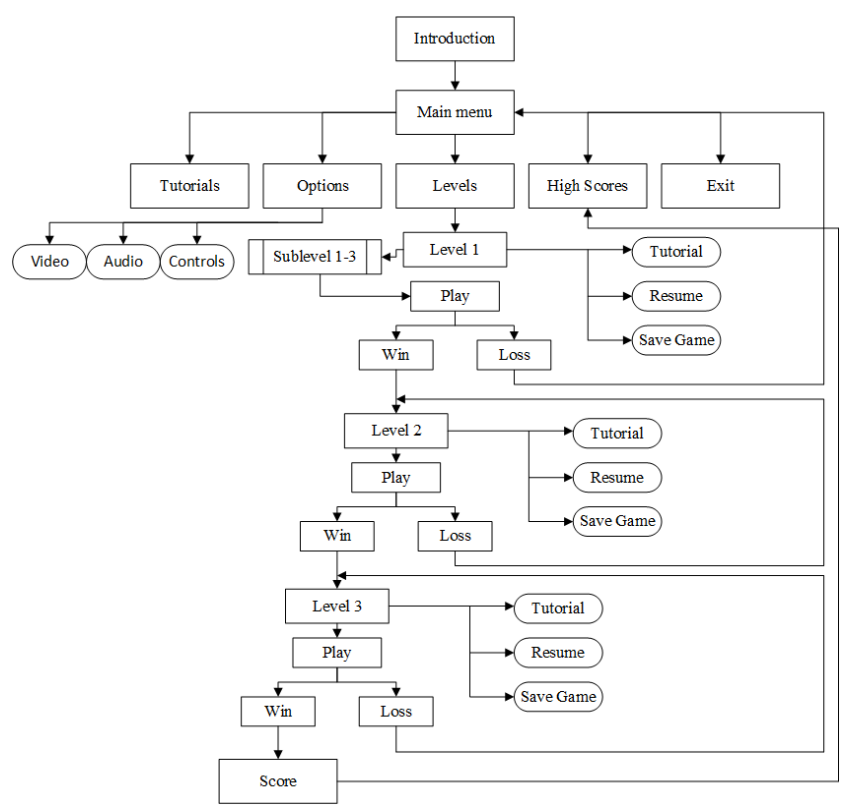

Fig. 1. Architecture of the game.

This level includes three sublevels. Sublevel 1 will identify organs and indicate their names, sizes, major functions and locations (see Figure 2). Sublevel 2 will identify structures, functions, and diseases of organs. Sublevel 3 will outline treatments of diseases of these organs. These sublevels can be unlocked and played after the player has correctly answered all questions in the respective areas. These levels are designed to provide a much more interactive and entertaining environment for the player to explore human models in depth, while at the same time embracing traditional game play elements. All of this is combined to create a disguised learning environment in which the notion of learning is blended with a fun, interactive element.

Level 2 is built using a symptom-based approach. It presents both common and rare symptoms and outlines how they should be interpreted. This will help a practitioner achieve an accurate diagnosis in order to prescribe an appropriate treatment. At this stage, a traditional menu bar will be used, including clickable buttons that would lead the user to the selected screen. The intent is for the user to be able to learn about a human model within the game and apply the newly attained knowledge to other parts of the game.

Level 3 will help each individual to learn the diagnostic process in clinical medicine with a case-based approach (see Figure 3). The menu includes causes, diagnoses, treatments, complications, and preventative measures. This level will teach each user an evidence-based approach for evaluating, diagnosing, and treating patients based on their clinical complaints. The user will recognize a specific disease and prescribe the most effective therapy. Each case addresses one common complaint and guidance on how to organize the differential diagnosis. The case unfolds in a stepwise fashion as tests are performed and diagnoses are confirmed.

Unity was chosen to implement the prototype game. Unity is a cross-platform game creation system developed by Unity Technologies, which includes a game engine and integrated development environment. Unity is notable for its ability to target games to multiple platforms. Within a project, developers have control over delivery to mobile devices, web browsers, desktops, and consoles.

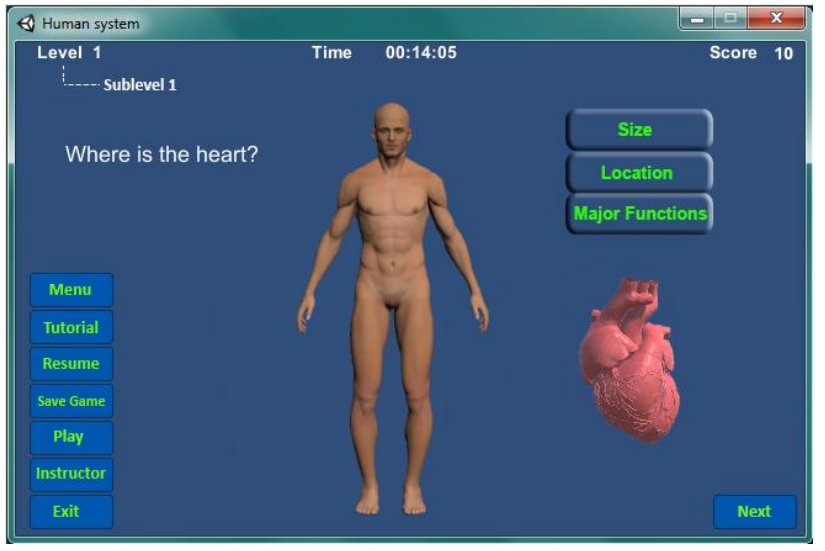

Fig. 2. Basic features of an organ listed in Sublevel 1.

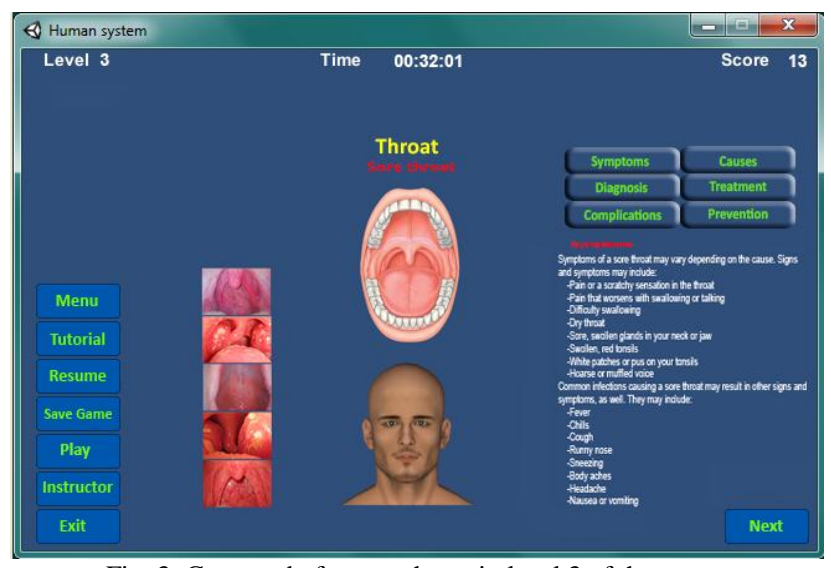

Fig. 3. Case study for sore throat in level 3 of the game.

\section{CONCLUSIONS}

The focus of this paper is the design and development of an educational game. The prototype covers all of these areas by enabling learners to explore and learn about human models within a virtual environment. The game provides different challenges for different players. Within the game, there are multiple levels and sub-levels where players can gradually make progress. While not all users may find the prototype entertaining, they will likely feel that there was a sufficient level of challenge. Providing education in an appealing way can be difficult, but many users believe that the presentation and enjoyable elements will ensure that they continue to play the game.

Future works include providing more difficult levels and the addition of more sublevels, as well as extending the game to $3 \mathrm{D}$ virtual reality in order to offer a more immersive experience to the users. Artificial intelligence could be incorporated into the game to make it more challenging as well. The game could also be used to target non-health professionals in order to increase their knowledge of healthcare-related topics. 


\section{ACKNOWLEDGMENTS}

This research is supported by National Science Foundation of China (61170324, 61572020).

\section{REFERENCES}

[1] M. Ma, A. Oikonomou, and L. C. Jain, Serious Games and Edutainment Applications, Springer, 2011.

[2] F. Laamarti, M. Eid, and A. E. Saddik, "An overview of serious games," International Journal of Computer Games Technology, 2014, 2014, p. 15.

[3] D. Michael and S. Chen, Serious Games: Games That Educate, Train, and Inform, Cengage Learning PTR, 2006.

[4] L. T. Kohn, J. M. Corrigan, and M. S. Donaldson, To Err Is Human: Building a Safer Health System, Washington, D.C.: National Academy Press, 2000.

[5] F. Ricciardi1 and L. T. D. Paolis, "A comprehensive review of serious games in health professions," International Journal of Computer Games Technology, 2014, p. 11.

[6] D. A. Cook et al., "Technology-enhanced simulation for health professions education: a systematic review andmeta-analysis," Journal of the American Medical Association, vol. 306, no. 9, pp. 978-988, 2011.

[7] H. W. R. Schreuder et al., "Implementation of simulation in surgical practice: Minimally invasive surgery has taken the lead: The Dutch experience," Medical Teacher, vol. 33, no. 2, pp. 105-115, 2011.

[8] A. S. Thijssen and M. P. Schijven, "Contemporary virtual reality laparoscopy simulators: Quicksand or solid grounds for assessing surgical trainees?" The American Journal of Surgery, vol. 199, no. 4, pp. 529-541, 2010.

[9] K. Henriksen and M. D. Patterson, "Simulation in health care: Setting realistic expectations," Journal of Patient Safety, vol. 3, pp. 127-134, 2007.

[10] J. B. Cooper and V. R. Taqueti, "A brief history of the development of mannequin simulators for clinical education and training," Quality and Safety in Health Care, vol. 13, pp. i11-i18, 2004.
[11] M. B. Jalink et al., "The effects of video games on laparoscopic simulator skills," The American Journal of Surgery, vol. 208, no. 1, pp. 151-156, 2014.

[12] C. V. Hove et al., "Predictors of technical skill acquisition among resident trainees in a laparoscopic skills education program," World Journal of Surgery, vol. 32, no. 9, pp. 1917-1921, 2008.

[13] I. Wenzler, Simulations as Learning from the Future, Develop, NR. 2, 2008, pp. 60-66.

[14] I. Mayer, "The research and evaluation of serious games: Toward a comprehensive methodology," British Journal of Educational Technology, vol. 45, no. 3, pp. 502-527, 2014.

[15] G. Hookway et al., "Learning physics through computer games," The 18th International Conference on Computer Games, 2013.

Alice J. Lin is interested in 3D computer simulation and animation, 3D computer modelling and algorithms, computer-aided geometric design, computer graphics, virtual reality, game design and development.

Michael Chen is interested in applying computer graphics and simulation to life sciences. He is also interested in database analysis and networking.

Fuhua (Frank) Cheng is a professor of computer science at the University of Kentucky, USA, and a joint professor of computer science at the Tsinghua University in Taiwan. He received his B.S. degree and M.S. degree in mathematics from the Tsinghua University in Taiwan, and his M.S. degree in computer science and Ph.D. degree in mathematics and computer science from the Ohio State University, USA. His research interests include computer aided geometric modeling, computer graphics, parallel computing in geometric modeling and computer graphics, and collaborative CAD. He is on the editorial board of Computer Aided Design \& Applications, Journal of Computer Aided Design \& Computer Graphics, Computer Aided Drafting, Design and Manufacturing and ISRN Applied Mathematics. 